\title{
Subtle signals at sea
}

\section{The ocean overturning circulation is potentially sensitive to climate change. In the north and south alike, human influence is less pronounced than we thought, but that is no reason to relax our watchfulness.}

Much of the ocean is deep, tucked well away from human observation. The bottom of the deepest ocean trench, for example, is more than 2,000 metres further away from sea level than the top of Mount Everest. Yet when we think about the sea, and its protection from human interference, the emphasis is usually on the upper layers of the ocean. Surface and abyss are connected, however, at a select number of locations of deep mixing (orange stars in the figure). With the July issue of Nature Geoscience, we present a web focus (http://go.nature.com/1UaNO99) that explores various facets of the ocean overturning circulation and its interactions with the climate system.

Vertical water exchange is largely limited to two broad centres of action: the northern North Atlantic and the oceans around Antarctica. Ocean dynamics in both regions can respond sensitively to changes in climate: deep vertical mixing is governed by seawater density, which is a function of temperature and salinity. The impacts of changes in vertical mixing and hence overturning could be both sudden and enormous. It is therefore not surprising that the notion of a weakening or even collapse of the ocean overturning circulation in response to warming or freshwater release has been on the palaeoceanography and climate science agendas for decades ${ }^{1}$. A wildly exaggerated version of this concept even made it into popular culture in the 2004 film The Day After Tomorrow: in the movie, the break-up of an Antarctic ice shelf is the harbinger of a full-scale collapse of the North Atlantic overturning circulation - which, in turn, plunges the entire Northern Hemisphere into a glaciated state in a matter of days.

Such cataclysmic catastrophe makes for good entertainment. But in the real world, the processes are much slower, and more subtle. A slow-down - let alone collapse - of the ocean overturning circulation is not readily discerned in the instrumental record, given the large amount of natural background variability. And attribution of any variations to anthropogenic climate change is even more challenging. Instead, two papers in this issue (on pages 513 and 518) come to the conclusion that the decline in North Atlantic overturning that has been observed since around 2004 reverses an invigoration over the preceding decade. Thus, the weakening

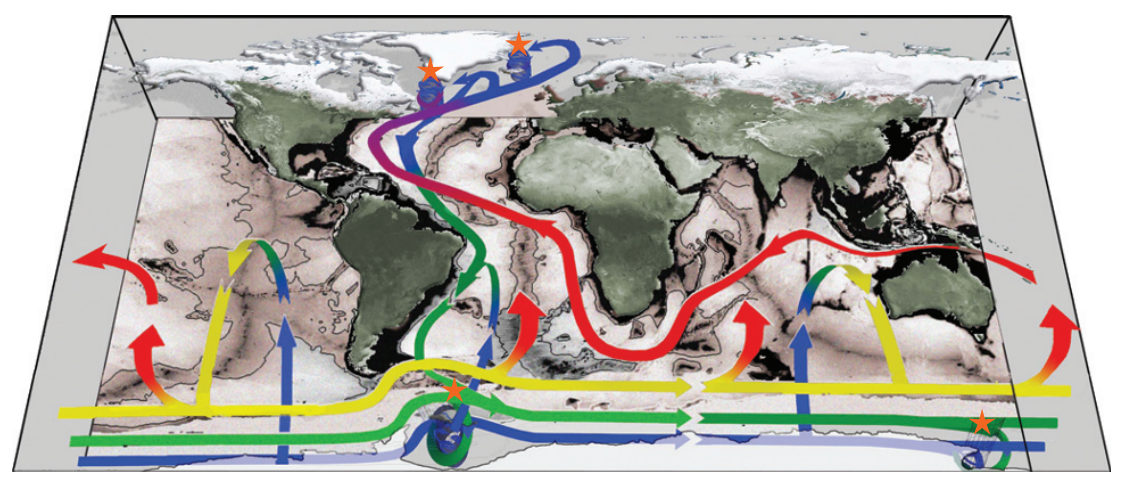

circulation in the past decade or so could well be part of a longer-term, decadalscale mode of variability rather than global warming-induced, at least so far. The RAPID array $^{2}-$ an array of instruments stretching across the Atlantic that measure temperature, salinity and current velocities - was, by chance, put in place in 2004, just when the circulation was at its peak. Now, looking back in time beyond 2004, the question of whether the observations point to human-induced circulation changes is wide open.

An influence of freshwater additions from the melting Greenland ice sheet on Atlantic overturning would point to human-induced climate warming as the culprit. However, such an influence cannot be pinned down at this point. On page 523 of this issue, Böning and colleagues note that although meltwater from Greenland has affected the salinity of surface waters in the Labrador Sea, these variations are still within the bounds of earlier salinity anomalies, and have not yet affected deepwater formation in this key location. In line with this picture, Castelao and colleagues note on page 528 that only a small fraction of the meltwater from southwest Greenland, where ice melt has increased fastest in the past few years, reaches the sites of deep overturning in the Labrador Sea.

It is important to monitor the ocean circulation closely. Multidecadal variations in the North Atlantic overturning circulation are a key link between atmospheric long-term variability and changes in other components of the Earth's climate system, such as Arctic sea ice, Northern Hemisphere temperature and Atlantic tropical storm activity, conclude Delworth and colleagues (page 509). That any changes so far seem to be consistent with natural variability is good news.
Over in the southern high latitudes, slow warming has been puzzling researchers. Overturning in the Southern Ocean is split into an upper and lower branch that together represent a key component of the global ocean circulation ${ }^{3}$. This southern overturning circulation is the reason for the sluggish pace of warming, argue Armour and colleagues on page 549. It brings up deeper water that has not yet been affected by human-induced climate warming and thereby keeps the region relatively cool. Nevertheless, palaeodata show that polar amplification - stronger climate warming at the highest latitudes - affects both poles in equal measure in the long run. Southern Ocean warming will reach its full potential more slowly, on timescales of centuries associated with changing deep-sea temperatures. It turns out that the freshwater fluxes during sea-ice formation and melting play a dominant role in governing the upper branch of the circulation ${ }^{4}$.

The picture emerges that a human influence on ocean overturning strength is elusive for now. Nevertheless, it may not be long before global warming changes the paths or vigour of the ocean circulation. We need to measure the reference state of the ocean now. And, if we want to stand a chance of being prepared we must be ready with our monitoring network on all available channels, by remote sensing and in situ observations, when anthropogenic change kicks in.

\footnotetext{
References

1. Broecker, W. S., Peteet, D. M. \& Rind, D. Nature 315, 21-26 (1985).

2. Smeed, D. et al. (British Oceanographic Data Centre-Natural Environment Research Council, 2015); http://dx.doi.org/10/6qb 3. Marshall, J. \& Speer, K. Nature Geosci. 5, 171-180 (2012).

4. Abernathey, R. P. et al. Nature Geosci. http://dx.doi.org/10.1038/ ngeo2749 (2016)
} 